\title{
Various Questions relative to the Triangle.
}

By Ed. Collignon.

1. If in a closed area $C D$ a point $O$ is given, and through this point a straight line $\mathrm{MN}$ is to be drawn such that the segment MCN so determined may be a minimum or a maximum, this condition is satisfied by drawing through $\mathrm{O}$ a chord MN having $\mathrm{O}$ for its mid point. Of the two segments which are separated by this chord and which are obtained by rotating the chord round $O$, the one MDN will be the maximum and the other MCN will be the minimum. The sum of the segments is equal to the whole area of the contour, and consequently if one of them corresponds to the minimum, the complementary segment corresponds to the maximum. The two segments interchange, the one into the other, when the chord $\mathrm{MN}$, turning round the point $\mathrm{O}$, undergoes a displacement equivalent to the angle $\pi$.

Apply what has preceded to a triangle $\mathrm{ABC}$, inside which a point $O$ is given.

Through $\mathrm{O}$ draw $\mathrm{OH}$ parallel to $\mathrm{AC}$ and meeting $\mathrm{CB}$ at $\mathrm{H}$; and make $\mathrm{H} m=\mathrm{CH}$. The straight line $m \mathrm{O} n$, whose mid point is $\mathrm{O}$ (Fig. 1), cuts off the minimum triangle $m C n$ (constructed within the angle $\mathrm{C}$ ), and the complementary area $m \mathrm{BA} n$ is the maximum.

In order that the extremities $m$ and $n$ of the chord inscribed in the triangle may be situated on the sides and not on the sides produced, it is necessary and sufficient that the segments $\mathrm{Cm}$ and Cn should be respectively less than the sides CB, CA or that their halves $\mathrm{CH}, \mathrm{HO}$ should be respectively less than the halves of these sides.

If therefore straight lines such as $m m$, drawn through the point $O$ within the three angles of the triangle $\mathrm{ABC}$, are to be inscribed in the triangle, that is to say, are to have their extremities situated on the sides and not on the sides produced, it is necessary and sufficient to take the point $O$ inside the triangle $A^{\prime} B^{\prime} C^{\prime}$ whose vertices are the mid points of the sides of triangle $A B C$. 
One can then construct the three straight lines (Fig. 2) $m n, p q, r \delta$ which have $O$ for common mid point, and which form within the angles $\mathrm{C}, \mathrm{A}, \mathrm{B}$ of the given triangle the triangles of minimum area. If $m p, r n, q s$ be drawn, new chords are obtained which are parallel and equal to the segments of the sides $n q, s m, p r$, and which complete the hexagon mprnqs inscribed in the triangle and having $O$ for its centre. The vertices of the hexagon will be situated on a curve of the second order also having the point $O$ for centre; and three systems of conjugate diameters of the curve will be obtained by joining the point $\mathrm{O}$ to the mid points of the sides $s m, p r, n q$, or to the mid points of the opposite sides $r n, q s, m p$. It will then be easy to trace the curve or to find its equation.

\section{Definition of the coordinates employed.}

2. We shall refer the point $O$ to the sides of the triangle taken as axes.

Let CB and CA be the axes adopted, the coordinates of any point being denoted by $x$ and $y$.

These coordinates will determine the position of the point $O$, or of any other point, with reference to the triangle, and we shall make use of them especially when we have to define the straight line $m n$ inseribed within the angle $C$.

For the other straight lines we shall use provisionally the coordinates $x^{\prime}, y^{\prime}$ referred to the axes $\mathrm{AB}, \mathrm{AC}$ if we are dealing with straight lines inscribed within the angle $A$; and the coordinates $x^{\prime \prime}, y^{\prime \prime}$ referred to the axes $\mathrm{BA}, \mathrm{BC}$ for the straight lines inscribed within the angle $B$.

The coordinates $x^{\prime}, y^{\prime}, x^{\prime \prime}, y^{\prime \prime}$ can easily be expressed in terms of the coordinates $x, y$.

For if $\mathrm{BC}, \mathrm{CA}, \mathrm{AB}$ be denoted by $a, b, c$

$$
\begin{array}{cl}
\frac{x^{\prime}}{x}=\frac{c}{a} & \text { or } x^{\prime}=\frac{c x}{a}, \frac{x^{\prime \prime}}{y}=\frac{c}{b} \quad \text { or } x^{\prime \prime}=\frac{c y}{b} \\
\frac{y+y^{\prime}}{b}=\frac{a-x}{a} & \text { or } y^{\prime}=\frac{a b-b x-a y}{a} \\
\frac{x+y^{\prime \prime}}{a}=\frac{b-y}{b} & \text { or } y^{\prime \prime}=\frac{a b-b x-a y}{b} .
\end{array}
$$


Areas of the triangles $m \mathrm{C} n$.

3. The triangle $m \mathrm{Cn}$ is double of the parallelogram (Fig. 1) CHOL which has for measure the product $x y$ sinC.

Hence, denoting by $\mathrm{T}(\mathrm{C})$ the area of triangle $m \mathrm{C} n$

Similarly

$$
\begin{aligned}
& T(C)=2 x y \quad \sin C \\
& T(A)=2 x^{\prime} y^{\prime} \sin A \\
& T(B)=2 x^{\prime \prime} y^{\prime \prime} \sin B .
\end{aligned}
$$

In the two last products substitute for the factors $x^{\prime}, y^{\prime}, x^{\prime \prime}, y^{\prime \prime}$ their values in terms of $x, y$; then

$$
\begin{aligned}
& \mathrm{T}(\mathrm{A})=2 \frac{c x}{a} \times \frac{a b-b x-a y}{a} \sin \mathrm{A} \\
& \mathrm{T}(\mathrm{B})=2 \frac{c y}{b} \times \frac{a b-b x-a y}{b} \sin \mathrm{B} \\
& \mathrm{T}(\mathrm{C})=2 x y \sin \mathrm{C} .
\end{aligned}
$$

Take the ratios of these three areas to the total area of the triangle which may be represented by the known formulæ

$$
\mathrm{T}(\mathrm{ABC})=\frac{1}{2} b c \sin \mathrm{A}=\frac{1}{2} c a \sin \mathrm{B}=\frac{1}{2} a b \sin \mathrm{C}
$$

and use for the total triangle the formula which eliminates the sines of the angles.

Then, if $\mathbf{R}(\mathrm{A}), \mathbf{R}(\mathrm{B}), \mathbf{R}(\mathrm{C})$ denote the three ratios

$$
\begin{aligned}
& R(A)=\frac{T(A)}{T(A B C)}=\frac{4\left(b a x-a x y-b x^{2}\right)}{b a^{2}} \\
& R(B)=\frac{T(B)}{T(A B C)}=\frac{4\left(b a y-b x y-a y^{2}\right)}{a b^{2}} \\
& R(C)=\frac{T(C)}{T(A B C)}=\frac{4 x y}{a b} .
\end{aligned}
$$

The angles $\mathrm{A}, \mathrm{B}, \mathrm{C}$ of the given triangle and the side $c$ of the same triangle disappear together, so that we shall obtain the same ratios $\mathbf{R}$ if we vary the angle $\mathbf{C}$ of the triangle whose sides serve us as coordinate axes. It will be seen that the algebraic expression of the three ratios contains only the ratios $\frac{x}{a}, \frac{y}{b}$ of the coordinates $x$ and $y$ to the sides on which they are counted. 
To simplify the expressions, put

The ratios then become

$$
\xi=\frac{x}{a} \quad \eta=\frac{y}{b} .
$$

$$
\begin{aligned}
& \text { (1) } \mathrm{R}(\mathrm{A})=4\left(\xi-\xi^{2}-\xi \eta\right) \\
& \text { (2) } \mathrm{R}(\mathrm{B})=4\left(\eta-\eta^{2}-\xi \eta\right) \\
& \text { (3) } \mathrm{R}(\mathrm{C})=4 \xi \eta .
\end{aligned}
$$

Between the three equations which we have just obtained eliminate $\xi$ and $\eta$. The final equation will be the relation which necessarily exists between the three ratios $\mathbf{R}(\mathbf{A}), \mathbf{R}(\mathbf{B}), \mathbf{R}(\mathbf{C})$ independently of the form and the dimensions of the triangle as well as the situation of the point $O$.

To make this elimination, put $R(C)$ for $4 \xi \eta$ in equations (1) and (2) ; then

$$
\begin{aligned}
& \text { (4). } \xi-\xi^{2}=\frac{R(A)+R(C)}{4} \\
& \text { (5) } \eta-\eta^{2}=\frac{R(B)+R(C)}{4} .
\end{aligned}
$$

From these two equations by addition and by subtracting half of equation (3), we have

$$
\begin{aligned}
\xi+\eta & -\left(\xi^{2}+2 \xi \eta+\eta^{2}\right)=(\xi+\eta)-(\xi+\eta)^{2} \\
& =\frac{\mathbf{R}(\mathrm{A})+\mathrm{R}(\mathrm{B})+2 \mathrm{R}(\mathrm{C})}{4}-\frac{\mathbf{R}(\mathrm{C})}{2} \\
& =\frac{\mathbf{R}(\mathrm{A})+\mathrm{R}(\mathrm{B})}{4} .
\end{aligned}
$$

From equations (4) and (5) by multiplication we have

$$
\begin{aligned}
\xi \eta- & \xi \eta(\xi+\eta)+\xi^{2} \eta^{2}=\frac{\mathbf{R}(\mathrm{A})+\mathbf{R}(\mathrm{C})}{4} \times \frac{\mathbf{R}(\mathrm{B})+\mathbf{R}(\mathrm{C})}{4} \\
& =\frac{\mathbf{R}(\mathrm{A}) \mathbf{R}(\mathrm{B})+\mathbf{R}(\mathrm{C})\{\mathbf{R}(\mathrm{A})+\mathbf{R}(\mathrm{B})\}+\mathbf{R}^{2}(\mathrm{C})}{16}
\end{aligned}
$$

an equation which, if we put $\frac{R(C)}{4}$ for $\xi \eta$, becomes

$$
\begin{aligned}
& \frac{R(C)}{4}-\frac{R(C)}{4}(\xi+\eta)+\frac{R^{2}(C)}{16} \\
& =\frac{R(A) R(B)+R(C)\{R(A)+R(B)\}+R^{2}(C)}{16} .
\end{aligned}
$$



have

The terms $\frac{\mathrm{R}^{2}(\mathrm{C})}{16}$ destroy each other, and solving for $\xi+\eta$ we

$$
\begin{aligned}
\xi+\eta & =\frac{1 \mathrm{R}(\mathrm{C})-\frac{1}{10}[\mathrm{R}(\mathrm{A}) \mathrm{R}(\mathrm{B})+\mathrm{R}(\mathrm{C})\{\mathrm{R}(\mathrm{A})+\mathrm{R}(\mathrm{B})\}]}{\frac{1}{4} \mathrm{R}(\overline{\mathrm{C}})} \\
& =1-\frac{\mathbf{R}(\mathrm{A})}{4}-\frac{\mathbf{R}(\mathrm{B})}{4}-\frac{\mathrm{R}(\mathrm{A}) \mathrm{R}(\mathrm{B})}{4 \mathrm{R}(\mathrm{C})} .
\end{aligned}
$$

If this value be substituted in equation (6) we have the relation sought :

$$
\begin{gathered}
\left(1-\frac{\mathrm{R}(\mathrm{A})}{4}-\frac{\mathrm{R}(\mathrm{B})}{4}-\frac{\mathrm{R}(\mathrm{A}) \mathrm{R}(\mathrm{B})}{4 \mathrm{R}(\mathrm{C})}\right)-\left(1-\frac{\mathrm{R}(\mathrm{A})}{4}-\frac{\mathrm{R}(\mathrm{B})}{4}-\frac{\mathrm{R}(\mathrm{A}) \mathrm{R}(\mathrm{B})}{4 \mathrm{R}(\mathrm{C})}\right)^{8} \\
=\frac{\mathrm{R}(\mathrm{A})+\mathrm{R}(\mathrm{B})}{4} .
\end{gathered}
$$

Now this relation is not in the definitive form which may be given to it, and which ought to be symmetrical with regard to the three ratios, for the three triangles play the same part in the given triangle.

To reach this form, begin by making symmetrical the parentheses of the first side. We may put

(11) $R(A)+R(B)+\frac{R(A) R(B)}{R(C)}=\frac{R(B) R(C)+R(C) R(A)+R(A) R(B)}{R(C)}$

and denote by $\mathrm{V}$ the numerator of the second side

$$
V=R(B) R(C)+R(C) R(A)+R(A) R(B)
$$

the sum of the products two by two of the three ratios. We shall then have

$$
\left(1-\frac{\mathrm{V}}{4 \mathrm{R}(\mathrm{C})}\right)-\left(1-\frac{\mathrm{V}}{4 \mathrm{R}(\mathrm{C})}\right)^{2}=\frac{\mathrm{R}(\mathrm{A})+\mathrm{R}(\mathrm{B})}{4} .
$$

Developing the square and simplifying we have

$$
\frac{V}{4 R(C)}-\frac{V^{2}}{16 R^{2}(C)}=\frac{R(A)+R(B)}{4} .
$$

But from equation (11)

$$
\frac{V}{4 R(C)}=\frac{R(A)+R(B)}{4}+\frac{R(A) R(B)}{4 R(C)}
$$


Hence equation (14) reduces to

$$
\frac{R(A) R(B)}{4 R(C)}-\frac{V^{2}}{16 R^{2}(C)}=0
$$

whence, multiplying by $16 \mathrm{R}^{2}(\mathrm{C})$

$$
\begin{aligned}
4 R(A) R(B) R(C) & =V^{2} \\
= & \{R(B) R(C)+R(C) R(A)+R(A) R(B)\}^{2}
\end{aligned}
$$

a formula symmetrical with respect to $R(A), R(B), R(C)$.

Developing the square and dividing by the first side we obtain a form somewhat simpler

$$
\frac{1}{4}\left\{\frac{R(B) R(C)}{R(A)}+\frac{R(C) R(A)}{R(B)}+\frac{R(A) R(B)}{R(C)}\right\}+\frac{R(A)+R(B)+R(C)}{2}=1 .
$$

\section{Examination of Particular Cases.}

\section{Verifications.}

4. Place the point $O$ at $G$ the centre of gravity of the triangle.

If through this point we draw parallels to the sides of the triangle, they will have their mid points at $G$ and will satisfy the conditions of the problem.

The three triangles $m \mathrm{C} n, p \mathrm{~A} q, r \mathrm{~B} s$ are equivalent in this particular case; they are similar to the given triangle; and consequently the ratios $R(A), R(B), R(C)$ are equal, and, the ratio of the sides being $\frac{2}{3}$, the ratio of the surfaces is $\frac{4}{9}$. The final equation (16) or (17) should therefore be satisfied by substituting this value for each of the ratios. We have

$$
\begin{array}{ll}
\text { form (16) } & 4 \times\left(\frac{4}{8}\right)^{3}=\left\{\left(\frac{4}{8}\right)^{2} \times 3\right\}^{2} \\
\text { form (17) } & \frac{1}{4} \times\left(\frac{4}{8} \times 3\right)+\frac{8}{2} \times \frac{4}{8}=1 .
\end{array}
$$

Take the point $O$ at $I$ the mid point of the side AC.

The finite line $\mathrm{AC}$ is, within the angle $\mathrm{ABC}$, the only straight line which is divided at the point I into two equal parts. This line is also the only limiting straight line having its mid point at $I$, and contained within the two angles ACB, CAB.

Relatively to the first signification of $A C$ we have $R(B)=1$, since the corresponding triangle is the triangle $A B C$ itself ; relatively to the two others we have $R(A)=R(C)=0$. These values verify the two forms of the general condition, 
If we take arbitrarily two ratios $\mathrm{R}(\mathrm{A}), \mathrm{R}(\mathrm{B})$, for example, the equation (16) will give the third ratio $R(C)$. It is of the second degree with respect to the unknown quantity, and consequently furnishes two values for the ratio sought; they will be of the same sign. Choose either of them. We may then construct the triangle $\mathrm{ABC}$, taking arbitrarily the two sides $a$ and $b$ and the angle $\mathrm{C}$ contained between them; the ratio $R(C)$ will be related to the triangle constructed within this angle $C$. We have then, making use of the coordinates $\xi$ and $\eta$, referred to the sides $a$ and $b$

$$
\begin{aligned}
& \xi+\eta=1-\frac{\mathrm{R}(\mathrm{B}) \mathrm{R}(\mathrm{C})+\mathrm{R}(\mathrm{C}) \mathrm{R}(\mathrm{A})+\mathrm{R}(\mathrm{A}) \mathrm{R}(\mathrm{B})}{4 \mathrm{R}(\mathrm{C})} \\
& \xi \eta=\frac{\mathrm{R}(\mathrm{C})}{4} .
\end{aligned}
$$

These coordinates will be given by the equation of the second degree

$$
t^{2}-\left\{1-\frac{\mathrm{R}(\mathrm{B}) \mathrm{R}(\mathrm{C})+\mathrm{R}(\mathrm{C}) \mathrm{R}(\mathrm{A})+\mathrm{R}(\mathrm{A}) \mathrm{R}(\mathrm{B})}{4 \mathrm{R}(\mathrm{C})}\right\} t+\frac{\mathrm{R}(\mathrm{C})}{4}=0
$$

and may be permuted, the one into the other.

In order that the problem may be possible it is necessary that the values of $\xi$ and $\eta$ be real ; it is necessary besides that the point defined by the coordinates

$$
x=a \xi \quad y=b \eta
$$

should be within the triangle $\mathrm{A}^{\prime} \mathrm{B}^{\prime} \mathrm{C}^{\prime}$, which is obtained by joining the mid points of the sides of the given triangle.

We must therefore have the inequalities

$$
\xi<\frac{1}{2}, \eta<\frac{1}{2}, \quad \xi+\eta>\frac{1}{2}
$$

which is equivalent to putting

$$
x<\frac{a}{2}, y<\frac{b}{2}, b x+a y>\frac{a b}{2} .
$$

If these inequalities are not verified, the constructions take place outside the triangle; and the formulæ still apply, but on the condition that suitable signs are given to the areas to be evaluated, according to the conventions of analytical geometry and the integral calculus. The curve passing through the vertices of the hexagon then becomes a hyperbola. 


\section{Area of the inscribed hexagon.}

5. Let us return to the principal case where (Fig. 2) the point $O$ is contained within the triangle $A^{\prime} B^{\prime} C^{\prime}$, and where the straight lines $m n, p q, r s$ determine the vertices of a hexagon inscribed in the triangle $\mathrm{ABC}$.

Let $\Omega$ be the area of this figure.

Its value will be obtained by subtracting from the area $\mathrm{S}$ of the triangle $\mathrm{ABC}$, the three triangles $\mathrm{Anr}, \mathrm{B} m p, \mathrm{C} q s$, formed within the angles $\mathrm{A}, \mathrm{B}, \mathrm{C}$ by the sides parallel to the sides of the given triangle, and which are consequently similar to this triangle. We have therefore

and hence

$$
\begin{aligned}
& \text { area } \mathrm{A} n r=\mathrm{S} \times\left(\frac{\mathrm{A} n}{\mathrm{AC}}\right)^{2}=\mathrm{S} \times\left(\frac{b-2 y}{b}\right)^{2} \\
& \text { area } \mathrm{C} q s=\mathrm{S} \times\left(\frac{\mathrm{C} q}{\mathrm{CA}}\right)^{2}=\mathrm{S} \times\left(\frac{b-2 y^{\prime}}{b}\right)^{2} \\
& \text { area } \mathrm{B} m p=\mathrm{S} \times\left(\frac{\mathrm{B} m}{\mathrm{BC}}\right)^{2}=\mathrm{S} \times\left(\frac{a-2 x}{a}\right)^{2}
\end{aligned}
$$

$$
\Omega=\mathrm{S}-\mathrm{S}\left\{\left(\frac{b-2 y}{b}\right)^{2}+\left(\frac{b-2 y^{\prime}}{b}\right)^{2}+\left(\frac{a-2 x}{a}\right)^{2}\right\} .
$$

In this equation substitute for $y^{\prime}$ its value in terms of $x$ and $y$, and for $\frac{x}{a}, \frac{y}{b}$ the proportional coordinates $\xi$ and $\eta$; then

$$
\begin{aligned}
\frac{b-2 y}{b} & =1-2 \eta \\
\frac{b-2 y^{\prime}}{b} & =\frac{b-\frac{2 a b-2 b x-2 a y}{a}}{b} \\
& =1-2(1-\xi-\eta)=2(\xi+\eta)-1 \\
\frac{a-2 x}{a} & =1-2 \xi
\end{aligned}
$$

and finally

$$
\begin{aligned}
\Omega & =\mathrm{S}\left[1-(1-2 \eta)^{2}-\{2(\xi+\eta)-1\}^{2}-(1-2 \xi)^{2}\right] \\
& =\mathrm{S}\left[8(\xi+\eta)-8\left(\eta^{2}+\eta \xi+\xi^{2}\right)-2\right]
\end{aligned}
$$

which may be written

$$
\xi+\eta-\left(\eta^{2}+\eta \xi+\xi^{2}\right)=\frac{\Omega}{8 \mathrm{~S}}+1 .
$$


If the area $\Omega$ is given in advance, or rather the ratio $\frac{\Omega}{\mathbf{S}}$, the equation (20) defines the curve along which the point $O$ must be taken in order that the corresponding hexagon may have the given area $\Omega$.

To verify the formula take successively

$$
\begin{array}{ll}
\xi=0 & \eta=\frac{1}{2} \\
\xi=\frac{1}{3} & \eta=\frac{1}{8} .
\end{array}
$$

Formula (20) gives for the first hypothesis $\Omega=0$; and for the second $\Omega=\frac{8 \mathrm{~S}}{12}=\frac{2}{3} \mathrm{~S}$, which agrees with the results already obtained.

The curve which gives to the area $\Omega$ a determinate value is an ellipse

$$
\eta^{2}+\xi \eta+\xi^{2}-\eta-\xi+1=\text { constant. }
$$

It has for centre the point

$$
\xi=\frac{1}{3} \quad \eta=\frac{1}{3}
$$

that is to say, the centre of gravity of the triangle.

If we vary the constant, all the curves are similar and have the point $G$, the centre of gravity of the triangle, for centre of similitude.

If we wish to know the maximum value of the area $\Omega$, it is sufficient to put equal to zero the partial derivatives of the function which represents the value of $\frac{\Omega}{8 \mathrm{~S}}$. This gives

$$
\begin{aligned}
2 \eta+\xi-1 & =0 \\
\eta+2 \xi-1 & =0
\end{aligned}
$$

that is, $\xi=\eta=\frac{1}{3}$, coordinates of the centre of gravity.

The hexagon is therefore a maximum for the point $G$, the common centre of the ellipses. At this point we have $\Omega=\frac{2}{3} \mathrm{~S}$, and the ellipse reduces to its centre.

If we put $\Omega=0$, the equation of the curve becomes

$$
\eta^{2}+\xi^{2}+\xi \eta-\eta-\xi+\frac{1}{4}=0
$$

which represents the ellipse passing through the mid points of the three sides and having these sides for tangents.

A similar ellipse can be inscribed in the triangle $\Lambda^{\prime} \mathrm{B}^{\prime} \mathrm{C}^{\prime}$. ( ( $\mathrm{HL}^{\prime}$ of the points of contact has for coordinates $\xi=\eta=1$, and conserpuently for all the points of this ellipse we shall lave $\Omega=! n$. 
Among these similar ellipses must be counted that which passes through the three vertices $A, B, C$. If we take the value of $\Omega$ for the vertex $C$ as origin, we shall have

and consequently $\frac{\Omega}{8 \mathrm{~S}}+\frac{1}{4}=0$

$$
\xi=\eta=0
$$

whence $\Omega=-2 \mathrm{~S}$.

We can express the area $\Omega$ as a function of the ratios $\mathbf{R}(\mathrm{A}), \mathbf{R}(\mathrm{B}), \mathbf{R}(\mathrm{C})$.

For we have

$$
\begin{gathered}
\xi \eta=\frac{R(C)}{4} \\
\xi+\eta=1-\frac{1}{4}\left\{R(A)+R(B)+\frac{R(A) R(B)}{\mathrm{R}(\mathrm{C})}\right\} \\
\eta^{2}+\xi \eta+\xi^{2}=(\eta+\xi)^{2}-\xi \eta \\
=\left[1-\frac{1}{4}\left\{\mathbf{R}(\mathrm{A})+\mathbf{R}(\mathrm{B})+\frac{\mathrm{R}(\mathrm{A}) \mathrm{R}(\mathrm{B})}{\mathrm{R}(\mathrm{C})}\right\}\right]^{2}-\frac{\mathrm{R}(\mathrm{C})}{4}
\end{gathered}
$$

and equation (20) becomes

$$
\begin{aligned}
1- & \left\{R(A)+R(B)+\frac{R(A) R(B)}{R(C)}\right\} \\
& -\left[1-\frac{1}{4}\left\{R(A)+R(B)+\frac{R(A) R(B)}{R(C)}\right\}\right]^{2}+\frac{R(C)}{4}=\frac{\Omega}{8 S}+\frac{1}{4}
\end{aligned}
$$

Since we have transformed the first side into $\frac{R(A)+R(B)}{4}$, by equation (13) we have

that is

$$
\frac{\Omega}{8 \mathrm{~S}}+\frac{1}{4}=\frac{\mathrm{R}(\mathrm{A})+\mathrm{R}(\mathrm{B})+\mathrm{R}(\mathrm{C})}{4}
$$

$$
\begin{aligned}
\Omega & =8 S\left[\frac{R(A)+R(B)+R(C)}{4}-\frac{1}{4}\right] \\
& =2 S\{R(A)+R(B)+R(C)-1\} .
\end{aligned}
$$

